Title:

Design Study for a Superconducting Proton Linac From 20 to $100 \mathrm{MeV}$

Author(s):

T. Wangler, R. Garnett, K, Krawczyk, J. Billen, N. Bultman, K. Christensen, W. Fox, and R. Wood

Submitted to:

1993 Particle Accelerator Conference, May 17-20, 1993, Washngton, D.C.

Los Alamos National Laboratory, an affirmative action/equal opportunity employer, is operated by the University of California for the U.S. Department of Energy under contract W-7405-ENG-36. By acceptance of this article, the publisher recognizes that the U.S. Government retains a nonexclusive, royalty-free license to publish or reproduce the published form of this contribution, or to allow others to do so, for U.S. Government purposes. The Los Alamos National Laboratory requests that the publisher identify this article as work performed under the auspices of the U.S. Departmont of Energy. 


\title{
DESIGN STUDY FOR A SUPERCONDUCTING PROTON LINAC FROM 20 TO 100 MEV*
}

\author{
T. P. Wangler, R. Gamett, F. Krawczyk, J. Billen, N. Bultman, \\ K. Christensen, W. Fox, and R. Wood \\ Los Alamos, National Laboratory, Los Alamos, NM 87545
}

\section{INTRODUCTION}

Advances in superconducting radiofrequency technology during the past 15 years have made possible the large-scale application of superconducting niobium accelerators. So far this development has been restricted to rather low-current electron and heavy-ion accelerators. In addition to the power savings, the improved capability of superconducting cavities to provide acceleration of high currents with low beam losses, which follows from the ability to use larger beam apertures without a large economic penalty from increased if losses, could make superconducting proton linacs very attractive for high-intensity applications, where activation of the accelerator is a major concern. During the past year, at Los Alamos, we have been looking at a possible upgrade to the $800-\mathrm{MeV}$ LAMPF proton accelerator, to provide higher intensity injection into a new storage ring for a new high-intensity pulsed neutron source. As part of this upgrade to the LAMPF accelerator, the entire linac below $100 \mathrm{MeV}$ would be rebuilt to provide improved beam quality, improved reliability, and to include funneling at $20 \mathrm{MeV}$ for higher beam currents. Both a roomtemperature and a superconducting option are being considered for the section from 20 to $100 \mathrm{MeV}$. At present, this section is a $201.25 \mathrm{MHz}$ room-temperature copper drifttube linac (DTL). For this new upgrade scenario the frequency from 20 to $100 \mathrm{MeV}$ was fixed at $805 \mathrm{MHz}$. The new duty factor is assumed to be $7.2 \%$, and we will show some results at two currents, $30 \mathrm{~mA}$ and $150 \mathrm{~mA}$, that span the range of interest. Our superconducting linac concept consists of individual multicell cavities, each driven by a klystrode. Focusing would be provided by superconducting quadrupole lenses between cavities. In the remainder of the paper we describe our study to evaluate the potential of a superconducting proton linac section for this application, and address some of the many design choices.

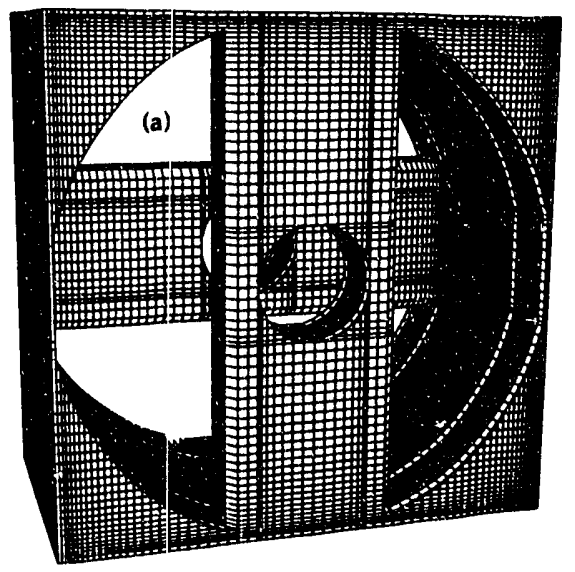

\section{ACCELERATING STRUCTURE STUDIES}

Recently, at Argonne, a new njobium accelerating structure, called the spoke resonator ${ }^{1}$ has been built and tested at high fields. This structure is suitable for acceleration of high-current beams at the intermediate velocities of interest. This structure is also equivalent to the slotted iris structure that was built many years ago for superconducting applications at Karlsruhe 2 . The cavity that was tested resonated at $855 \mathrm{MHz}$, and was optimized for a particle velocity of $0.30 \mathrm{c}$. RF-tests were performed on the cavity and a high accelerating gradient of $7.2 \mathrm{MV} / \mathrm{m}$, limited by magnetic quench, was obtained at a $4.2 \mathrm{~K}$ operating temperature. A peak surface electric field of $24 \mathrm{MV} / \mathrm{m}$ and a peak surface magnetic field of $560 \mathrm{G}$ were obtained in the cavity. The accelerating gradient achieved in the test is already very attractive for a proton linac application.

For a proton linac it may be more economical to employ accelerating structures with more than two gaps. We have looked at two arrangements for the spokes, a) the ladder geometry where all the spokes are parallel, and b) the crossbar geometry, where adjacent spokes are rotated by 90 degrees. We have studied the characteristics of both these structures at $805 \mathrm{MHz}$ and at three different velocities, corresponding to 20,60 , and $100 \mathrm{MeV}$ protons, using the 3D electromagnetic code MAFIA(Release 3.1). Figure 1 shows drawings produced by MAFIA for each of these structures. For these initial studies the spoke has been modeled as a rectangular bar of width $=6.0 \mathrm{~cm}$ with beveled edges to reduce the peak electric field, and thickness $=0.317$ $L_{c}$ along the axial direction, where $L_{c}=\beta \lambda / 2$ is the cell length, and $\beta$ and $\lambda$ are the velocity and if wavelength. The boundary conditions are defined for MAFIA on the transverse planes that pass through the center of the bars. For the $\pi$ mode the electric fields are parallel to the boundary

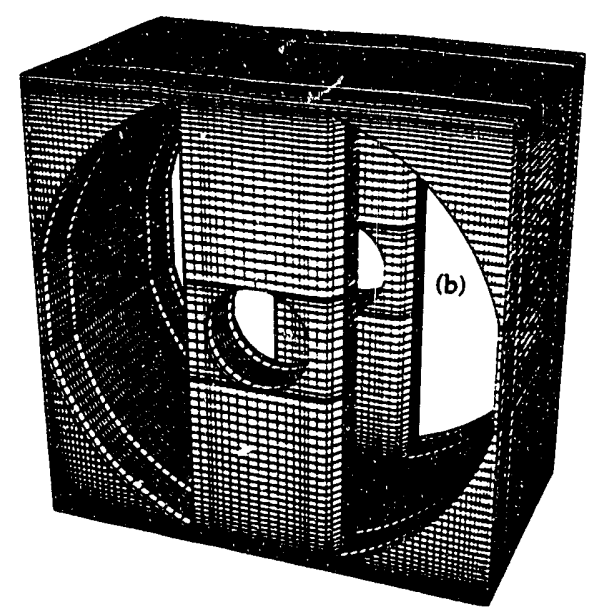

Fig. 1. Views of the multicell cavity structures designed for particles with velocity $=0.428 \mathrm{c}$ produced by MAFIA. a) Crossbar structure, b) Ladder structure.

\footnotetext{
*Work supported by Los Alamos National Laboratory Institutional Supporting Researches under the auspices of the United States Department of Energy.
} 
plane. The cavity radius $R$ was adjusted until the resonant frequency of the $\pi$ mode was within $1 \%$ of $805 \mathrm{MHz}$. The results are shown in Table 1, where the cross-bar and ladder structures are designated as $C B$ and $L$. The notation is: $Q_{0}$ is the unloaded quality factor, $R_{S}(\Omega)$ is the rf surface resistance, $T$ is the transit-time factor, $k=\left(\omega_{\pi}-\omega_{0}\right) / \omega_{\text {ave }}$ is the intercell coupling factor defined in terms of the frequencies of the 0 and $\pi$ modes, and their average frequency, $U(\mathrm{~mJ})$ is the stored energy within a length $\beta \lambda$, $E_{p}(M V / m)$ is the peak surface electric field, and $B_{p}(m T)$ is the peak surface magnetic field. The stored energy and field values are normalized so that the accelerating field $\mathrm{E}_{0} \mathrm{~T}=1$ $\mathrm{MV} / \mathrm{m}$, where $E_{0}$ is the spatial average of the axial electric field. The coupling factor $k$ is larger for the CB case but it is probably acceptably large for both structure types.

The peak surface field differences between the two structures are the result of 3D geometry effects. The peak surface electric fields generally occur at the edges of the bar even when the edges are beveled. For the $\mathrm{CB}$ structure the electric field lines that originate from the face of a bar tend to terminate preferentially along the edges of the adjacent perpendicular bar, and this concentration produces an enhanced peak surface field. Improvements in the spoke geometry should reduce Ep. The peak surface magnetic field is maximum where the bar intersects the cylindrical wall. For the CB structure the current and the magnetic field are not smoothly distributed around the bar, but are concentrated along the sides. For the $C B$ structure this results in the shortest path for the current. Although the optimization of the geometries is not completed, the smaller peak surface fields for the $\mathrm{L}$ structure makes this structure an attractive candidate.

We have also looked at the conventional 0-mode DTL structure. A disadvantage of the 0 -mode structure is that the transit-time factor is reduced, because the axial field leaks into the drift tubes, when large apertures are required. We have considered an example in which the drift tubes will not contain a focusing quadrupole, and are designed with noses to compensate for the reduction in the transit-time factor caused by the large aperture. The DTL has been modeled as an azimuthally symmetric structure, and it's properties have been calculated using the 2D electromagnetic code SUPERFISH. The results are also shown in Table 1. By comparison with the spoke-type structures, the DTL also has very strong intercell coupling, lower $T$, much larger $E_{p}$, and smaller $B_{p}$ (but see footnote for Table 1).

\section{BEAM DYNAMICS STUDIES}

We present two examples ${ }^{3}$ from our study of superconducting linac solutions using the code CCLDYN. The multicell cavities are generated for a fixed value of velocity $\beta$, all cells in the cavity have the same length $\beta \lambda / 2$, and each cavity has a different $\beta$. The intertank spaces are chosen to allow adequate room for flanges, bellows, diagnostics, and quadrupole lenses. The beam current, averaged over an rf cycle, is assumed to be either 30 or $150 \mathrm{~mA}$. The accelerating field $E_{0} T$ as a function of beam energy was obtained from a polynomial fit to MAFIA calculations, and corresponds approximately to a constant peak surface electric field near $20 \mathrm{MV} / \mathrm{m}$. We chose $\mathrm{E}_{0} \mathrm{~T}$ equal to 3.5 $\mathrm{MV} / \mathrm{m}$ at $20 \mathrm{MeV}, 5.1 \mathrm{MV} / \mathrm{m}$ at $60 \mathrm{MeV}$, and $5.5 \mathrm{MV} / \mathrm{m}$ at $100 \mathrm{MeV}$.

The two linac designs we have considered correspond to a 2.0-cm radial aperture and a fixed energy gain per multicell cavity equal to a) $\Delta \mathrm{W}=1.0 \mathrm{MeV}$, and b) $\Delta \mathrm{W}=3.33 \mathrm{MeV}$. The number of cells per cavity is determined by $\Delta W, E_{0} T$, and the synchronous phase. Table 2 summarizes the parameters for the two examples. We interpret the results as follows. For design B the linac is shorter and has fewer components. However, for design B: a) the cavity input power coupler requirements are greater (The present state of the art for input power couplers represents a limit that is generally considered to be about $100 \mathrm{~kW}$.), and b) the transverse aperture-to-rms beam size ratio is lower as a result of the reduced transverse phase advance per focusing period, leading to a larger beam envelope and indicating increased beam-loss potential. We consider that for $150 \mathrm{~mA}$ the transverse aperture to $\mathrm{rms}$ ratio is probably more than adequate for design $A$, and the longitudinal ratio is more than adequate for design $B$. In these designis with quadrupoles between all cavities, increasing the cavity energy gain and length increases the quadrupole period, which for fixed $80^{\circ}$ transverse phase-advance per period, reduces the overall quadrupole focusing strength. However, it improves the overall longitudinal focusing, because of the reduced number of intertank spaces. The beam dynamics optimum in cavity energy gain lies somewhere between the two designs. Additional optimization would include the choices of aperture and synchronous phase.

It may be desireable from the point of view of reliability to restrict the number of gaps per cavity to only 2 or 3 , so that failure of a single cavity would result in only a minor perturbation on the overall linac performance. We have not had time to explore this issue, but we note that the multicell cavities for the two designs presented here could be replaced by groups of two or three gap cavities. Thebeam dynamics results for this case should be similar to what has

Table 1 MAFIA results for cross-bar(CB) and ladder(L) structures in the $\pi$ mode and DTL structure in 0 mode.

$\begin{array}{lllllllll}\text { Type } & \beta & \mathrm{R}(\mathrm{m}) & \mathrm{Q}_{0} \mathrm{R}_{\mathrm{s}}(\Omega) & \mathrm{T} & \mathrm{k} & \mathrm{U}(\mathrm{mJ}) & \mathrm{E}_{\mathrm{p}}(\mathrm{MV} / \mathrm{m}) & \mathrm{B}(\mathrm{mT})^{*} \\ \text { CB } & 0.207 & 0.0745 & 63.7 & 0.782 & 0.58 & 16.9 & 6.16 & 24.9 \\ \mathrm{CB} & 0.341 & 0.0845 & 85.0 & 0.776 & 0.46 & 17.6 & 4.14 & 16.4 \\ \mathrm{CB} & 0.428 & 0.0891 & 93.3 & 0.771 & 0.40 & 21.4 & 3.86 & 14.6 \\ \mathrm{~L} & 0.207 & 0.0981 & 62.5 & 0.782 & 0.31 & 18.8 & 5.09 & 19.4 \\ \text { L } & 0.341 & 0.1000 & 86.5 & 0.777 & 0.30 & 18.3 & 3.58 & 13.6 \\ \text { L } & 0.428 & 0.1003 & 93.9 & 0.779 & 0.30 & 21.0 & 3.43 & 13.0 \\ \text { DTL } & 0.207 & 0.10 & 207.9 & 0.498 & 0.90 & 23.6 & 20.5 & 8.16 \\ \text { DTL } & 0.341 & 0.10 & 213.6 & 0.641 & 0.61 & 23.2 & 14.4 & 7.20 \\ \text { DTL } & 0.428 & 0.10 & 210.1 & 0.633 & 0.51 & 32.0 & 14.2 & 8.09\end{array}$

* For the DTL this does not include the factor of 2 stem enhancement. 
Table 2 Comparison of $805 \mathrm{MHz}, 20-100 \mathrm{MeV}$ Superconducting Linac Designs

PARAMETER

Energy gain per cavity (MeV)

$\mathrm{E}_{0} \mathrm{~T}(\mathrm{MV} / \mathrm{m})$

Synchronous Phase (deg)

Cells per Cavity

Number of Cavities

Cavity Lengths (m)

Cavity Input Power (kW)

Intercavity Spacing

Aperture Radius $(\mathrm{cm})$

Transverse Aperture to Rms Beam-Size Ratio

Longitudinal Aperture to Rms Beam-Size Ratio

Focusing Lattice Type

Total Length(m)

been already presented in this paper, provided that the gaps required between cavities are small.

In Table 3 we show various power values at operating temperatures of both $2 \mathrm{~K}$ and $4.2 \mathrm{~K}$. The peak rf power of 2.4 MW is determined by the beam power for an assumed 30 $\mathrm{mA}$ beam current. The average rf power is obtained by applying the $7.2 \%$ duty factor. The rf surface resistances are taken from measurements at $805 \mathrm{MHz}$ on single-cell niobium elliptical cavities ${ }^{4}$ at $E p=20 \mathrm{MV} / \mathrm{m}$, and the surface resistance at $2 \mathrm{~K}$ is lower than at $4.2 \mathrm{~K}$ by a factor of 16.7 . The rf power dissipation in the niobium structure has been obtained by using the parameters from the MAFIA calculations for the $L$ structure. The static heat leak is estimated ${ }^{5}$ at $7 \mathrm{~W} / \mathrm{m}$ of cryostat length, and the length is taken from the design A value of $62 \mathrm{~m}$. Because the static heat leak is a continuous load, while the rf load is pulsed, the static effect is dominant at $2 \mathrm{~K}$ and is comparable to the of load at $4.2 \mathrm{~K}$. For this reason the large temperature dependence in the if surface resistance does not produce a correspondingly large difference in the thermal power into the Helium. The refrigeration-efficiency ${ }^{6}$ difference favors the $4.2 \mathrm{~K}$ temperature, mostly because of the temperature dependence of the Carnot efficiency factor. The net result is that the ac refrigeration power is about equal for the two operating temperatures. This conclusion would change in favor of $2 \mathrm{~K}$ operation, if the accelerating field increased, if the duty factor increased, or if the static heat load decreased.

Table 3 Superconducting Linac Power Values

$\begin{array}{lll}\text { Operating Temperature } & 2 \mathrm{~K} & 4.2 \mathrm{~K} \\ \text { Beam current (mA) } & 30 & 30 \\ \text { Peak rf power(MW) } & 2.4 & 2.4 \\ \text { Average rf power (kW) } & 170 & 170 \\ \text { Niobium rf surface resistance (n } \Omega) & 24 & 400 \\ \text { Peak rf power-niobium (W) } & 520 & 8600 \\ \text { Average rf power-niobium(W) } & 37 & 620 \\ \text { Static heat leak power (W) } & 430 & 430 \\ \text { Total power into Helium(W) } & 470 & 1050 \\ \text { Refrigeration efficiency } & 1 / 1200 & 1 / 530 \\ \text { AC power for refrigeration(kW) } & 560 & 560 \\ \text { AC klystrode power(kW) } & 370 & 370 \\ \text { Total ac power }(\mathrm{kW}) & 930 & 930\end{array}$

$\begin{array}{ll}\text { DESIGN A } & \text { DESIGN B } \\ 1.00 & 3.33 \\ 3.5 \text { to } 5.5 & 3.5 \text { to } 5.5 \\ -40 \text { to }-35, & -40 \\ 9 \text { to } 3 & 30 \text { to } 9 \\ 87 & 26 \\ 0.18 \text { to } 0.36 & 0.71 \text { to } 1.18 \\ 30(30 \mathrm{~mA}) & 100(30 \mathrm{~mA}) \\ 150(150 \mathrm{~mA}) & 500(150 \mathrm{~mA}) \\ 5.5 \beta \lambda \text { to } 3.5 \beta \lambda & 5.5 \beta \lambda \text { to } 3.5 \beta \lambda \\ 2.0 & 2.0 \\ 14-25(30 \mathrm{~mA}) & 7-14(30 \mathrm{~mA}) \\ 11-19(150 \mathrm{~mA}) & 6-10(150 \mathrm{~mA}) \\ 6-9(30 \mathrm{~mA}) & 12-13(30 \mathrm{~mA}) \\ 5-6(150 \mathrm{~mA}) & 10-11(150 \mathrm{~mA}) \\ \text { Doublet } & \text { Doublet } \\ 62.0 & 36.9\end{array}$

The ac power for the klystrodes is calculated using an efficiency factor ${ }^{\gamma}$ of $47 \%$. The final conclusion is that at 30 $\mathrm{mA}$ the peak $\mathrm{if}$ power required is $2.4 \mathrm{MW}$, and the total ac operating power (refrigeration plus klystrodes) is just under $1 \mathrm{MW}$. The superconducting linac sections would reduce the peak rf power by $7.0 \mathrm{MW}$ and the ac operating power by 0.8 MW, compared to the existing $201.25 \mathrm{MHz}$ roomtemperature copper DTL.

\section{ACKNOWLEDGMENTS}

One of us, T.P.W., wishes to acknowledge several very productive discussions with Jean Delayen and Cort Bohn from Argonne on superconducting linac issues. Discussions with Los Alamos colleagues Lloyd Young, George Spalek, Brian Rusnak, Joe Dimarco, Ed Gray, Mike Lynch, Don Reid, and Andy Jason have been very important. We would like to thank Bob Jameson, Andy Jason, and Jim Stovall for their support in carrying out this work.

\section{REFERENCES:}

[1] J. R. Delayen, W. L. Kennedy, and C. T. Roche, "Design and Test of a Superconducting Structure for High-Velocity Ions", Proceedings of the 1992 Linear Accelerator Conference, Ottawa, Ontario, Canada, 2428 August, 1992, AECL Report AECL-10728, p 695.

[2] K. Mittag, "Superconducting Accelerator Structures for Medium Energy Protons", IEEE Trans. Nucl. Sci., Vol. NS-24, No. 3, 1156 (1977).

[3] R. W. Garnett, "Example Beam Dynamics Solutions for a 20-100 MeV Superconducting Linac for LANSCEII", Los Alamos Accelerator Technology Division Technical Note No. AT-1:93-099, April 15, 1993.

[4] Brian Rusnak, Los Alamos, private communication.

[5] C. H. Rhode and D. Proch, "Cryogenic Operation for Cavity Systems", Proc. of 4th Workshop on RF Superconductivity, KEK Report 89-21, KEK Laboratory, Tsukuba, Japan, August 14-18, 1989, p 751.

[6] P. Lebrun, "Cryogenic Systems", Superconductivity in Particle Accelerators< CERN Accelerator School Proceedings, CERN 89-04, Hamburg, Germany, May 30-June 3, 1988, p 66.

[7] Mike Lynch, Los Alamos, private communication. 

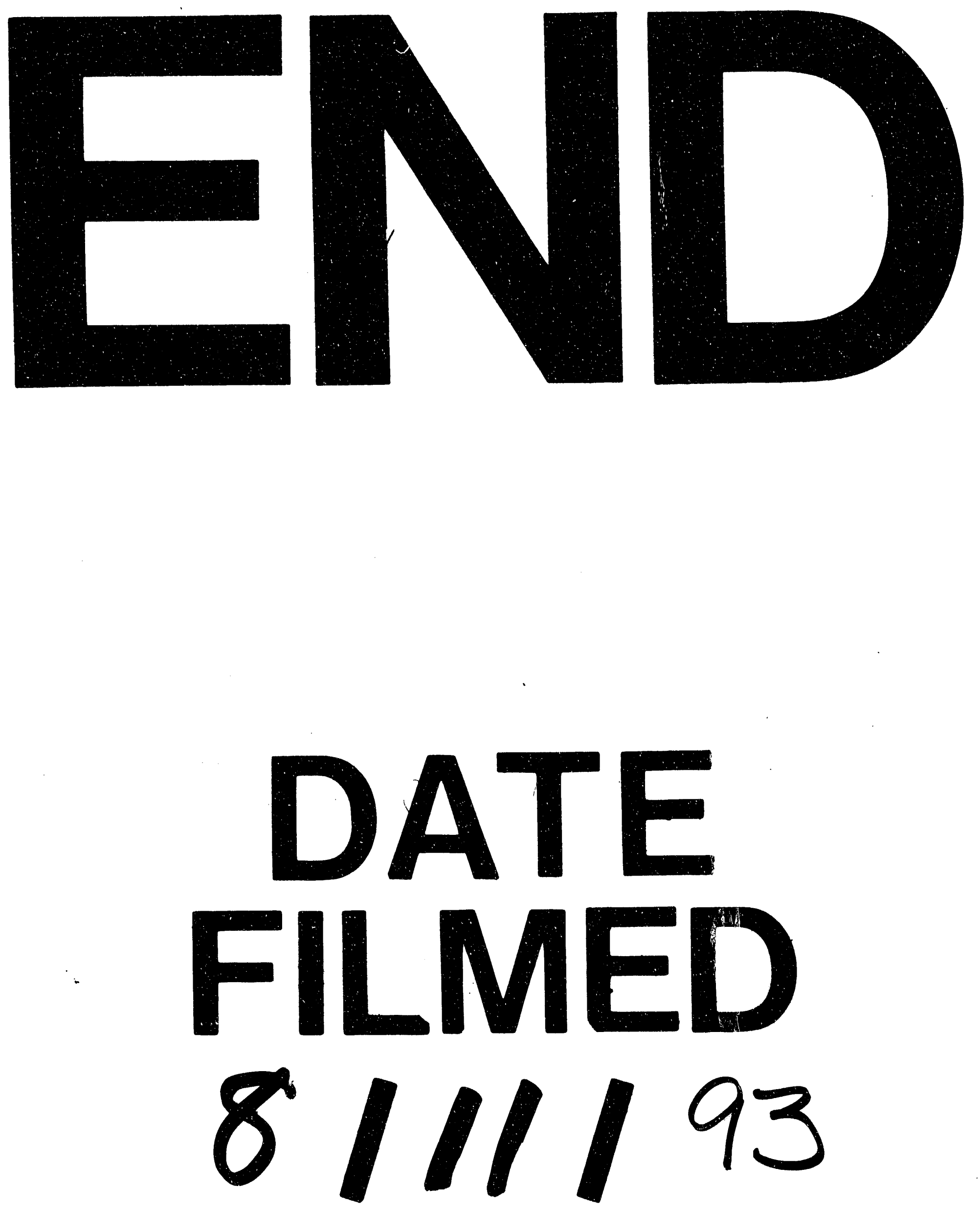
: 